\title{
Tangence
}

\section{Notes brèves sur la vistesse en science-fiction}

\section{Richard Saint-Gelais}

Numéro 55, septembre 1997

La vitesse

URI : https://id.erudit.org/iderudit/025952ar

DOI : https://doi.org/10.7202/025952ar

Aller au sommaire du numéro

Éditeur(s)

Tangence

ISSN

0226-9554 (imprimé)

1710-0305 (numérique)

Découvrir la revue

Citer cet article

Saint-Gelais, R. (1997). Notes brèves sur la vistesse en science-fiction. Tangence, (55), 138-153. https://doi.org/10.7202/025952ar d'utilisation que vous pouvez consulter en ligne.

https://apropos.erudit.org/fr/usagers/politique-dutilisation/ 


\section{Notes brèves sur la vitesse en science-fiction Richard Saint-Gelais}

Ce mouvement de désir demeurait au fond un désir de mouvement, comme il arrive quand on ne peut se mouvoir vers quelque endroit parce que le monde n'existe pas [...]

Italo Calvino

La vitesse, c'est de toute évidence l'affaire de ce genre pressé qu'est la science-fiction. La Lune est inaccessible? Qu'à cela ne tienne: il suffit d'imaginer un moyen, plaisant ou plausible - fioles emplies de rosée, obus tiré par un gigantesque canon ${ }^{1}$ - et le tour est joué. Les étoiles se trouvent à des distances telles que des centaines de vies humaines, mises bout à bout, ne suffiraient pas pour les atteindre? Peu importe: un savant a inventé un moyen de propulsion suffisamment rapide pour que les explorateurs y arrivent en moins de temps qu'il nous en faut pour nous extraire d'un embouteillage. La réalité suivra bien un jour, mais la science-fiction sera alors loin devant, impatiente toujours. Décrivant un fantasme exemplairement science-fictionnel, Theodore Sturgeon raconte quelque part ${ }^{2}$ que, lorsque les stations orbitales seront chose courante, il se trouvera encore des écrivains pour ne pas se contenter de situer leurs intrigues dans leur monde (futur), pour plutôt en anticiper d'autres, plus éloignés encore du nôtre. Conclusion de Sturgeon: "When I think of that, I want to live forever." Frustration devant la lenteur désespérante du réel, mais aussi belle illustration de cet auto-enchâssement ouvert auquel le genre semble se prêter avec une jubilation particulière: le fantasme de Sturgeon est à la fois une méditation sur la science-fiction des temps futurs et un petit récit de science-fiction (au deuxième degré si l'on veut), qui saute par-dessus les siècles que ni Sturgeon ni ses lecteurs ne

\footnotetext{
1 On aura reconnu, respectivement, Les Estats et empires de la Lune de Cyrano de Bergerac et De la Terre à la Lune de Jules Verne.

2 Theodore Sturgeon, "Introduction", dans New Soviet Science Fiction, New York, Macmillan, 1979, p. xi.
} 
vivront pour ainsi déboucher sur un futur au carré, esquissé déjà, noir sur blanc, dans toute sa vertigineuse béance.

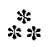

On répondra que ce court-circuit n'est que discursif, et que le futur en question demeure sans substance. Ce serait toutefois ne pas apercevoir qu'il n'en va jamais autrement: les descriptions les plus luxuriantes ne livrent - ne construisent - pas autre chose que des simulacres de planètes ou de sociétés futures. Tout comme les technologies science-fictionnelles de la vitesse (réacteurs tachyoniques, corridors de l'hyperespace, etc.) relèvent d'un tecbno-babble dont l'effet de séduction tient en partie à sa plausibilité scientifique - effective ou supposée - en partie au tour de passe-passe textuel qu'il opère. Pouvoirs de la science, pouvoirs du texte: la vitesse est peut-être le lieu où ces deux incommensurables s'imbriquent le plus étroitement, jusqu'à devenir inextricables aux yeux des amateurs. Passer d'un secteur à l'autre de la galaxie? Quelques heures y suffisent - quelques phrases aussi, que le lecteur traverse à la faveur d'un équivalent discursif de l'hyperespace: un espace où l'on quitte temporairement le fil de l'intrigue, mais où quelque principe étrange donne l'impression d'être expliqué, par le narrateur lui-même ou par un personnage obligeant:

Dans les nouveaux vaisseaux gravitiques, il était tout simplement impossible de remarquer le moment où le décollage avait lieu. Il n'y avait pas d'effet d'inertie, pas de poussée d'accélération, pas de bruit, pas de vibration.

Le Far Star pouvait s'isoler des champs gravitationnels extérieurs jusqu'au degré souhaité; il s'élevait au-dessus d'une surface planétaire comme s'il se mettait à flotter sur une mer cosmique. Pendant ce temps, paradoxalement, le champ gravitationnel à l'intérieur du vaisseau demeurait normal.

Bien entendu, tant que le vaisseau demeurait dans l'atmosphère, aucune accélération n'était nécessaire, de telle sorte qu'on ne sentait pas le bourdonnement et la vibration causés par le passage rapide à travers l'air. Au-delà de l'atmosphère, cependant, l'accélération pouvait commencer, et même atteindre un taux considérable, sans affecter les passagers. ${ }^{3}$

3 Isaac Asimov, Foundation and Earth, New York, Del Rey, 1987 11986l, p. 35. Ma traduction. 
“Ensuite M. Spock n'a qu'à pénétrer dans le réservoir, et à se tenir dans n'importe quelle position; puis on referme l'écoutille et on active la machine. Il ne s'apercevra de rien, car même s'il se retrouvera temporairement dans un espace à $n$ dimensions, ses sens ne lui permettent de percevoir que quatre dimensions à là fois, comme nous tous. Mais il ne disparaîtra pas - il ressortira aussitôt du réservoir. Pendant ce temps, sa réplique sera projetée vers Organia, et sera ramenée ici automatiquement, une journée après la matérialisation, quel que soit le moment où celle-ci aura eu lieu. Si ce délai ne suffit pas, nous recommencerons la manœuvre. Au moment où la réplique reviendra, nous aurons établi un espace de Hilbert dans le réservoir, qu'il suffira de maintenir jusqu'à ce que la réplique vienne faire son rapport .

"Tout cela me paraît suffisamment clair", dit Kirk. "M. Spock, êtes-vous prêt?" 4

- Nous avons un problème ici à la salle des machines ", continua l'ingénieur, qui sentait presque les systèmes de l'immense vaisseau se mettre en sommeil les uns après les autres. "Un problème très sérieux, capitaine.

- Quelle est la nature de ce problème, M. Scott?" demanda la voix de Spock, où ne perçait aucune émotion.

Scott avala sa salive. Il venait de se rendre compte qu'une sueur froide recouvrait son front, comme une éruption soudaine. "La valve de régulation du flux matière/antimatière a été laissée ouverte. Nous devons laisser l'alimentation au minimum, et couper complètement la propulsion de clistorsion, jusqu'à ce que nous ayons vérifié quelle est exactement la panne. "5

Vaisseaux gravitiques, annulation locale des champs gravitationnels, absence paradoxale d'effets pour les passagers, projection dans un espace à $n$ dimensions, dématérialisation et rematérialisation, espaces de Hilbert en guise de comité d'accueil, flux de matière/antimatière, propulsion de distorsion: la vitesse science-fictionnelle est toute discursive, ou plutôt lexicale: les astronefs carburent au vocabulaire. Il suffit de jouer le jeu. Peu importe que celui-ci soit truqué, et que, par exemple, les espaces hilbertiens, pour peu qu'on prenne la peine d'aller y voir de plus

4 James Blish, Spock Must Die!, New York, Bantam, 1970, p. 17. Ma traduction.

5 Della Van Hise, La croisée des temps, tr. fr. de Rosalic Guillatume, Paris, Arena, 1991 |1985|, p. 49. 
près, se révèlent, comment dire, peu propices aux déplacements intersidéraux :

On appelle espace vectoriel préhilbertien (complexe) un espace vectoriel sur le corps $\mathrm{C}$ des nombres complexes, muni d'une forme sesquilinéaire auto-adjointe dont la forme hermitienne associée est positive, c'est-à-dire une application de $\mathrm{E} x \mathrm{E}$ dans $\mathrm{C}$, notée $(x, y) \mid \rightarrow(x \mid y)$, satisfaisant aux conditions suivantes:

- pour tout élément y de $\mathrm{E}$, l'application $\mathrm{x} \mid->(x \mid y)$ est linéaire;

- pour tout couple $(x, y)$ d'éléments de $\mathrm{E},(y \mid x)=(\mathrm{x}+\mathrm{y})$;

- pour tout élément $\mathrm{x}$ de $\mathrm{E},(x \mid x) \geq 0$.

Le scalaire $(x \mid y)$ s'appelle produit hermitien des vecteurs $x$ et $y$.

On dit que l'espace vectoriel $\mathrm{E}$ est préhilbertien séparé, ou bermitien, si la forme hermitienne considérée est définie positive, c'est-à-dire si la relation $(x \mid x)=0$ implique la relation $x=0$.

[...]

En algèbre, on utilise surtout les espaces hermitiens de dimension finie. En analyse, ce sont surtout les espaces hermitiens de dimensions infinie qui interviennent dans la plupart des questions; on est amené à supposer que ces espaces sont complets, c'est-à-dire que toute suite de Cauchy est convergente. Un espace hermitien complet est dit bilbertien. Tout espace hermitien de dimension finie est hilbertien. ${ }^{6}$

On aura beau jeu d'ironiser, et d'imaginer la pauvre réplique de Spock saisie, au moment de se matérialiser, d'une crainte subite: les suites de Cauchy seront-elles bien convergentes? les techniciens auront-ils veillé à ce que la forme hermitienne associée soit positive? Il serait non moins facile d'opposer le discours scientifique, dont les termes ne peuvent être utilisés que s'ils ont été définis au préalable, à un discours science-fictionnel qui, parasitaire, n'en garderait que l'écume lexicale - aux charmes d'autant plus attrayants que le texte fait l'économie des aspérités de la démonstration. On n'en conclura pas pour autant à une opposition entre un discours de vérité qui serait celui de la science, et un discours fumiste, celui de la science-fiction: les mathématiques se soucient moins d'équivaloir au réel que de

6 L. Chambadal et J.-L. Ovaert, article "Hilbert (espace de)", Encyclopcedia Universalis, 1980, vol. 8, p. 393-394. 
142

construire, selon leurs règles propres, des objets qui ne sont peutêtre pas si dissemblables des objets fictifs:

Comme le platonicien et contrairement au formaliste, le mathématicien constructif reconnaît une certaine réalité aux objets mathématiques, mais les différencie essentiellement des objets matériels, en ne leur attribuant que les propriétés susceptibles de démonstration. Une distinction analogue différencie les héros de roman des personnages historiques. Une question concernant Vercingétorix admet une réponse, même si elle échappe à nos moyens d'investigation; la même question concernant Don Quichotte n'a pas de réponse si celle-ci ne peut être déduite des affirmations du roman de Cervantes [...] Don Quichotte est un être essentiellement incomplet. ${ }^{7}$

Mais il y a incomplétude et incomplétude. Celle des objets mathématiques (et plus généralement des concepts scientifiques) est une incomplétude opératoire: hors ce qui peut être dit mathématiquement d'un espace hilbertien, il n'y a rien à en dire. L'incomplétude des objets science-fictionnels est un peu autre: la référence à l'espace de Hilbert dans Spock Must Die! s'appuie sur un discours mathématique (qui, lui, indique ce qu'il en est de cet espace): discours absent, que peu de lecteurs iront consulter (et encore moins confronter à l'usage romanesque du concept), mais censé cautionner celui que le lecteur a sous les yeux ${ }^{8}$. Le discours science-fictionnel présuppose donc un discours d'une autre allure, qu'il ne tient pas - la science-fiction s'intéresse plus souvent qu'autrement aux mots de la science, pas à sa syntaxe ni à son discours - et vit tout entier de l'idée qu'il est toujours possible de délaisser temporairement le texte pour des régions discursives qu'en même temps on se garde bien d'aborder. La sciencefiction n'a cure des détours. Ou, plus exactement, elle multiplie les détours - ceux que constituent, par rapport à l'intrigue, les segments pseudo-scientifiques -, mais des détours qui sont autant de raccourcis: la quincaillerie lexicale de la science doit au

7 Roger Apéry, "Mathématiques constructives" dans François Guénard et Gilbert Lelièvre (dir.), Penser les mathématiques, Paris, Seuil, coll. "Points sciences ", 1982, p. 63-64.

8 Il ne faut toutefois pas sous-estimer la part de jeu que cela implique: il n'est pas du tout sûr que les lecteurs mesurent la crédibilité du roman à une (éventuelle) confirmation de sa justesse scientifique - et qu'ils suspendent entre-temps tout jugement à ce sujet. Aussi, des lecteurs sceptiques pourront, même en doutant de la plausibilité effective des explications fournics par le roman, accepter celles-ci comme des prémisses de l'univers fictif. 
plus vite déboucher sur l'usage diégétique qui en est fait; le récit regagne vite ses droits, à commencer par celui de se poursuivre.

$$
* * *
$$

S'agissant de plausibilité scientifique, l'obstacle est bien entendu la vitesse de la lumière, et la limite infranchissable - en principe - qu'elle impose aux déplacements, fussent-ils fulgurants. En faire fi reviendrait à ne pas jouer le jeu de la vraisemblance scientifique, à rompre l'équilibre délicat au profit de ce refoulé majeur de la science-fiction bard core: le texte. Aussi s'agit-il de contourner l'obstacle en prétendant exposer une solution qu'en fait on invente; ou, mieux encore, en présupposant une explication, censément disponible dans l'encyclopédie fictive, mais dont le texte fait l'économie: "Tandis que les sifflements des moteurs plasmatiques du cargo montaient de plusieurs tons, que sa massive carcasse frémissait sous la poussée, Balung, le copilote, observa avec anxiété le basculement de l'astéroïde dans le pano" ". Solution tributaire de l'évolution même de la science-fiction: comme le note Bud Foote, les innovations thématiques assimilées par le genre (par exemple, la machine à voyager dans le temps) finissent par se passer de l'encombrant appareil didactique qui les accompagnait à l'origine, et qui, bien entendu, ralentissait la progression de l'intrigue ${ }^{10}$. Cette solution offre aussi l'avantage de respecter l'exigence rationaliste, tout en évitant d'exhiber une "explication" qu'il serait trop facile de reconnaitre pour ce qu'elle est, à savoir un colmatage pseudo-scientifique: l'explication est bien fournie quelque part, dans un texte, mais celui-ci, ouvrage de physique ou manuel d'astronautique du $\mathrm{XXIV}^{\mathrm{e}}$ siècle, n'est pas celui qu'on lit. Dispositif à la Borges, ici au service d'une illusion référentielle passablement retorse.

$$
\text { *** }
$$

9 Jaan-Marc Ligny, Un été à Zedong, Paris, Fleuve noir, coll. "Anticipation", 1991 , p. 14. Je souligne.

10 Bud Foote, The Connecticut Yankee in the Tuentieth Century. Travel to the Past in Science Fiction, New York, Greenwood Press, 1991, p. 3. 
144

Vitesse du son, vitesse de la lumière: formules désormais banales, évidentes, qui nous dissuadent parfois de songer qu'il n'en a pas toujours été ainsi - que la lumière, et le son, ont déjà paru choses sans vitesse. Il aura fallu réfléchir un peu à partir du phénomène de l'écho, et du décalage qu'il induit, pour s'aviser que du temps se glisse entre cri et écoute. Assez curieusement, c'est encore par la réverbération qu'on est parvenu à mesurer la vitesse de la lumière - et à prouver, du coup, qu'elle en a une. On connaît le dispositif ingénieux imaginé par le physicien Albert Michelson au début du $\mathrm{xx}^{\mathrm{e}}$ siècle: obliger un faisceau lumineux à être réfléchi successivement par des miroirs disposés face à face qui accroissaient d'autant la distance franchie, et permettaient donc d'établir, entre départ et arrivée, une durée suffisamment longue pour être mesurable ${ }^{11}$. Je ne peux malgré tout m'empêcher de penser que Michelson n'est parvenu à mesurer la vitesse de la lumière qu'en la réduisant; j'imagine, tout à fait irrationnellement, une lumière ralentie, fatiguée presque, par tous ces allerretour - comme si la lumière acquérait, en même temps qu'une vitesse, une lenteur.

\section{***}

Il n'en est rien, bien sûr: la vitesse de la lumière est une constante $^{12}$, et le dispositif ne fait que démultiplier la distance qu'elle a à parcourir. La lumière n'en a pas moins une lenteur, et même une lenteur absolue et non relative: la lumière n'est pas lente par rapport à un autre phénomène qui serait plus rapide, mais lente parce que ramenée soudain, à rebours de l'intuition, du domaine de la fulgurance pure à celui du quantifiable. La science-fiction bard core, en un sens, n'en demande pas davantage: une fois que la lumière a une vitesse, celle-ci peut être pos-

11 Je simplifie quelque peu le dispositif imaginé par Michelson, qui comportait un miroir octogonal soumis à une rotation extrêmement rapide (approximativement 32,000 tours par minute). Pour plus de détails sur ses expériences (ainsi que celles de ses prédécesseurs Olaüs Roemer et Christiaan Huygens), voir David Martindale, Robert W. Heath et Philip C. Eastman, Principes fondamentaux de la physique. Cours avancé, traduction française de Luc Mainnemare, Montréal, Guérin, 1992 [1986], p. 407-410.

12 Du moins dans le vide; la vitesse de la lumière diminue selon le milieu (air, eau, verre, ctc.) à travers lequel elle se propage. 
tulée dépassable, par exemple par quelque particule comme les tachyons, opportunément "découverts" - découverte en fait purement conjecturale - à la fin des années 1960. Utiliser des particules inobservables, à la masse imaginaire (au sens mathématique du terme), à des fins de propulsion? Peu importe que l'idée apparaisse, dans les faits, irréalisable, voire grotesque: les "tachyons" iront grossir le lexique de la science-fiction, et l'arsenal des moyens de propulsion intersidérale.

La science-fiction, pour tout un chacun, c'est le rayon "histoires du futur". On a beau savoir que les choses ne sont pas aussi simples - il n'y a pas de futur, ou presque, chez Jules Verne, sans compter qu'un sous-genre entier, l'uchronie, est consacré non pas à l'avenir mais à la refabrication du passé -, il n'en clemeure pas moins que la science-fiction fait la part belle à l'anticipation ${ }^{13}$. Or celle-ci peut être abordée sous l'angle de la vitesse, si l'on considère qu'un texte d'anticipation gagne du temps en affichant sans plus tarder ce que l'Histoire, elle, produira lentement. À ce jeu, toutes les euphories sont permises: il suffit de dire qu'on y est pour se retrouver en 2889 , au dix-septième millénaire, en l'an un million - toutes époques qu'on viendra meubler de paysages technologiques ou sociaux dont la vraisemblance, on le sait maintenant, tient surtout aux horizons (ou aux obsessions) épistémologiques et idéologiques de l'époque de rédaction $^{1 / 4}$. Si la science-fiction nous a aidés à penser le présent

13 On notera toutefois que l'anticipation, qui nous est aujourd'hui tellement coutumière qu'elle nous semble aller (littérairement) de soi, est un motif romanesque qui ne s'est imposé qu'à la suite d'une lente mutation épistémique. Sur cette question, on consultera avec profit l'ouvrage de Paul K. Alkon, Origins of Futuristic Fiction, Athens, University of Georgia Press, 1987.

14 Fn ce sens, cette science-fiction parle au présent, dans les deux sens que prend cette expression. Que les temps grammaticaux qu'elle utilise relèvent massivement du passé, voilà qui donne un indice des périls qu'elle doit conjurer si elle veut imposer l'illusion d'une autonomie des futurs qu'elle décrit: l'emploi du passé n'est-il pas l'un des plus simples moyens d'accréditer l'idée que les événements décrits sont indépendants par rapport au discours et à ses conditions d'énonciation? Mais on notera aussi que l'emploi du passé pour relater des événements situés dans un futur lointain permet aussi de 
146

comme un futur en émergence, elle s'est vite sentie obligée, en retour, de réduire ce futur à l'explicitation de ce que le présent commençait, déjà, à faire affleurer. Il aura fallu un bon demisiècle pour que cette conception prospectiviste de l'anticipation soit sérieusement contestée.

On n'oubliera pas pour autant les vertus rhétoriques de l'anticipation: a beau mentir qui vient de loin, a beau spéculer qui situe loin. Or, de la même manière que les espaces intersidéraux sont volontiers escamotés par les télescopages techno-textuels, les vastes périodes intercalaires entre l'aujourd'hui de l'écriture et celui de la fiction ont généralement tendance à être expédiées en quelques paragraphes, voire en quelques lignes. Nulle Histoire alors, nul tissu complexe de transformations entre société présente et société future; tout cela est court-circuité au profit d'un confortable face à face entre deux époques liées essentiellement par des dispositifs argumentatifs, par exemple du type "If things go on like tbis, then...", qui réduisent le présent à une seule des lignes idéologiques qui traversent concurremment le discours social: la surpopulation, ou l'effet de serre, ou les expériences génétiques...

Or, ce face à face entre futur et présent (sensible, notamment, dans les textes où un avenir lointain est comparé... au $\mathrm{xx}^{\mathrm{C}}$ siècle ${ }^{15}$ ) peut être vu comme une force d'inertie, qui vient ralentir le bond fulgurant à travers les époques - quand il ne rappelle pas, par inadvertance, que ce futur n'est qu'un pur simulacre qui s'écrit et se lit aujourd'bui.

Les écrivains et lecteurs de science-fiction ont déjà prisé ce quasi-genre apparemment mineur, mais toujours spectaculaire, qu'on parvient difficilement à désigner mieux qu'à l'aide de son

projeter d'un scul coup la narration dans un futur encore plus lointain, comme le montre l'ingénieux City de Clifford D. Simak (New York, Ace Books, 1952).

15 Pour mesurer ce que cela a de curieux, on n'a qu’à imaginer un narrateur (jui se sentirait obligé de préciser, lorsqu'un personnage se saisit d'un téléphone, à quel point cette invention aurait sidéré un Italien de la Renaissance. 
nom anglais: sbort sbort story ${ }^{16}$. La short short story (ou s. s. s. pour les - improbables - intimes) n'est certes pas l'apanage de la science-fiction: on n'oubliera pas de sitôt, par exemple, "Le plus proche village", ni le labyrinthe minuscule et inextricable que disposent ses neuf lignes ${ }^{17}$. Mais, outre le fait que la plupart des s. S. s. relèvent probablement de la science-fiction ou du fantastique, il faut noter que la conjonction s. s. s. / sf (désolé!) a ceci de particulièrement saisissant qu'elle travaille à user du plus petit nombre de mots pour "couvrir", comme il est de règle en science-fiction, rien de moins qu'un monde imaginaire. Le pari est de taille, mais il a été tenu, à la faveur d'acrobaties qui font le charme, quand on l'éprouve, de ce petit genre. Un seul exemple, mais peut-être l'un des meilleurs: "Le choix" de W. HiltonYoung ${ }^{18}$, où il est question de ce qui constitue selon toute vraisemblance la première expédition temporelle vers l'avenir:

Avant de partir pour le futur, Williams s'acheta un appareil photo, un magnétophone et apprit la sténo. Cette nuit-là, quand tout fut prêt, nous fîmes du café et sortîmes du cognac en prévision de son retour.

Va pour l'appareil photo, pour le magnétophone et la sténo: Williams compte évidemment ramener autant d'informations que possible sur le monde futur. Par contre, la remarque sur le café pourra déconcerter, jusqu'à ce qu'on comprenne que, si Williams restera pendant une durée indéterminée dans le futur, rien ne l'empêche de revenir quelques minutes, voire quelques secondes

16 On pourrait bien sûr parler de "courte nouvelle", mais cette appellation manque de précision en ce qu'elle ne suggère pas forcément, me semble-t-il, le clegré de concision auquel s'astreint la short sbont story, qui serait plutôt une "micro-nouvelle, (voire une "nano-nouvelle "...). En outre, "shoit short story" offe l'avantage paradoxal d'accentuer la brièveté de la nouvelle, tout en donnant à penser que cette brièveté ne suffit pas à caractériser le genre: pourquoi redire de la nouvelle qu'elle est courte si elle n'est que cela? (Voir André Belleau, "Pour la nouvelle", dans André Carpentier, Du pain des oiseaux, Montréal, VLB éditeur, 1982, p. 10.) Sinon, la short sbort story serait un genre en forme de pléonasme, ce qui serait d'ailleurs assez piquant compte tenu qu'il faille en dire plus pour désigner un genre qui, pour sa part, se pirjue d'en dire le moins possible.

17 Fran\% Kafka, "Le plus proche village" dans La métamorphose, tr. fr. d'Alexandre Vialatte, Paris, Gallimard, coll. "Folio", 1955, p. 151.

18 W. Hilton-Young, "Le choix", tr. fr, de Bernard Raison, dans Demètre Ioakimidis, Jacques Goimard et Gérard Klein (dir.), Histoires paradoxales, Paris, Le Livre de Poche (coll. "La grande anthologie de la science-fiction", deuxième série), 1984, p. 113-114. 
148

seulement après être parti - juste à temps pour boire le cafécognac préparé entre-temps. On y reviendra: ce micro-épisode n'est pas sans rapport avec la vitesse.

On s'attachera pour l'instant à une autre vitesse, perpendiculaire en quelque sorte: celle du texte à qui quelques lignes, quelques notations sibyllines sur le café et le cognac suffisent pour mettre en place ce que la lecture, elle, devra déplier, selon une inférence qui ressemblera plus ou moins à celle que je viens de formuler (on imagine ici le lecteur lever la tête, "quitter le texte" pour examiner ses replis inférentiels avant de poursuivre son parcours, de reprendre le fil d'un texte où aucune durée ne se serait écoulée, tout comme Williams quitte le présent pour y revenir à la fois beaucoup plus tard - pour lui - et l'instant d'après - pour son collègue.)

Tout se passe comme prévu, à un détail près: Williams revient effectivement aussitôt, mais sans photos, sans enregistrements, sans notes, sans souvenirs - ou presque:

"Mais, juste ciel, protestai-je, pourquoi? Comment est-ce arrivé?

Tu ne te souviens donc de rien du tout?

- Je me souviens juste d'une chose.

- De quoi?

- On m'a tout fait voir et on m'a laissé le choix de m'en souvenir ou pas après mon retour.

- Et tu as choisi de ne pas t'en souvenir? Mais quelle idée incroyable t'a pris [sic] de...

- N'est-ce pas? répondit-il. On se demande bien pourquoi. "

Fin de la nouvelle - mais bien sûr pas de la lecture, manifestement conviée à imaginer quelles horreurs futures ont pu motiver la décision de Williams; invitée, donc, à se complaire dans un jeu d'extrapolations, tout en sachant que le texte n'en cautionnera ni n'en démentira aucune, jamais. Dispositif exemplaire que celui-là, qui parvient à se ranger sous la bannière de la science-fiction sans rien décrire d'un monde imaginaire, là (diégétiquement) sans être là (textuellement), tout entier confié aux aptitudes fabulatoires du lecteur. Texte fulgurant, mais d'une manière telle qu'il a de bonnes chances de provoquer cette lenteur par excellence de la lecture : la songerie. 
Voyager dans le temps? C'est ce que nous faisons tous, à la vitesse d'une seconde par seconde. Ceux pour qui cette vitesse est trop uniforme, ou qui ont envie de se mouvoir dans l'autre direction, n'ont guère le choix: ils doivent inventer une machine à voyager dans le temps, ou alors écrire de la science-fiction. On a abondamment glosé les paradoxes auxquels la chose peut donner lieu, pour peu que notre voyageur temporel élimine, par ignorance, inadvertance ou bravade, les conditions qui lui ont permis d'exister - c'est-à-dire, par exemple, tue son père ou sa mère avant que sa conception n'ait lieu ${ }^{19}$. On s'est moins intéressé aux aspects cinétiques du voyage temporel, qui sont pourtant, à bien y regarder, assez intrigants. Car ce qu'ouvre le voyage temporel, c'est la possibilité d'une vitesse d'une tout autre nature que celle à laquelle nous sommes habitués. Repensons à l'épisode du "Choix" pendant lequel on fait bouillir le café en attendant le retour de Williams: quelques minutes d'un côté, une durée indéterminée - mais certainement plus considérable - de l'autre. Les deux temporalités sont donc découplées, et une nouvelle vitesse doit être conçue: non plus une vitesse qui se mesurerait en fonction de la distance (parcourue) et du temps (écoulé), une vitesse $d / t$, mais bien plutôt une vitesse obtenue par la mise en rapport de $\operatorname{deu} x$ temps, une vitesse $t / t$, ou plus exactement $t i / t j$ : une vitesse où le temps se mesure au temps, où le temps n'est plus ce qu'il était. Ce à quoi le voyage temporel aboutit, c'est donc à une altération qualitative - et non plus seulement quantitative - de la vitesse.

\section{*:}

Mais ne délaissons pas trop vite les variations quantitatives: les écrivains de science-fiction, dont la sournoiserie cognitive ne doit jamais être sous-estimée, nous réservent de ce côté des surprises qui renouvellent, et pas peu, l'arsenal des paradoxes laissés par Zenon. Considérons par exemple la question suivante:

19 Comme cela arrive au héros du Voyageur imprudent de René Barjavel, sur lecpuel je dirai un mot plus loin. 
150

qu'est-ce qu'une vitesse extrême? On répondra: celle qui permet cle parcourir une distance $d$, considérable, en un temps $t$, si bref qu'il avoisine zéro. Mais ce serait compter sans ce sophiste en résidence de la science-fiction qu'est Robert Sheckley:

Avec sa ponctualité coutumière, Seethwright l'envoya sur une autre Terre type. la transition fut plus rapide que l'instantanéité. Elle fut si rapide, en fait, que le temps devint légèrement rétrograde et que Carmody eut la sensation étrange de réagir avant d'avoir reçu le stimulus nécessaire. C'était évidemment une contradiction, petite mais quand même illégale. Seethwright régla la chose à l'aide de la procédure d'oblitération standard, et personne ne prit la peine de mettre les autorités compétentes au courant. Les conséquences furent nulles à l'exception toutefois de l'usure du continuum spatio-temporel, dont Carmody ne s'aperçut même pas. ${ }^{20}$

Pourquoi, en effet, tenir le zéro pour une limite (au sens que le calcul intégral donne à ce terme)? Un déplacement vraiment rapide ne devrait-il pas prendre, non pas un temps infinitésimal, mais une durée négative - par exemple, moins sept secondes? Pourquoi se contenter d'une vélocité ordinaire, c'est-à-dire considérable, alors qu'on peut arriver à destination avant même d'être parti?

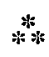

Les paradoxes temporels sont fréquemment pensés en termes logiques: enchevêtrement des causes et des effets, phénomènes d'auto-causalité, inversion du passé et de l'avenir. Mais ils peuvent aussi avoir des répercussions narratives, affectant le corps même du récit. Soit par exemple Le voyageur imprudent de Barjavel, dont tout le monde connaît le fameux dénouement circulaire: "Il [Saint-Menoux] a tué son ancêtre? Donc il n'existe pas. Donc il n'a pas tué son ancêtre. Donc il existe. Donc il a tué son ancêtre." 21 Pourtant, tout près, plus discrète (peut-être parce qu'aucune glose ne vient la souligner), gît une autre énigme,

20 Robert Sheckley, La dimension des miracles, tr. fr. de Guy Abadia, Paris, Laffont (réimpr. "Presses Pocket "), 1973, p. 195.

21 René Batravel, Le voyageur impmedent, Paris, Denoël (réimpr, "Folion), 1958. p. 243. 
aussi troublante: pourquoi Saint-Menoux, après être revenu du voyage temporel qui l'a mené en l'an 1793 (et, surtout, après avoir assassiné par erreur son ancêtre Durdat), met-il plusieurs heures à basculer dans cet état intercalaire entre existence et inexistence, d'où il ne sortira plus? En effet, puisque l'ancêtre est alors décédé depuis un siècle et demi, Saint-Menoux ne devrait-il pas subir les conséquences de son geste dès qu'il revient en 1943 - ou plutôt, dès qu'il revient au "1943" qui découle du passé désormais altéré, un "1943" où il n'a jamais vu le jour, faute de géniteur? Il n'en est pourtant rien: plusieurs pages raconteront ses agissements jusqu'au moment fatidique où il se "volatilisera": heures excédentaires, pages qu'en toute logique nous ne devrions pas lire. Il serait bien entendu facile, soit d'accuser Barjavel de négligence, soit de conclure que la délicate mécanique narrative du voyage temporel n'a été que progressivement maîtrisée par les écrivains de science-fiction - soit encore de voir dans ce ralentissement une manière de dramatiser la disparition (en soi peu spectaculaire, parce qu'instantanée) de Saint-Menoux. Mais une autre explication peut être avancée: sachant que le récit des dernières heures de Saint-Menoux alterne avec celui de l'agonie cle l'ancêtre, on peut se demander si notre voyageur temporel ne profite pas d'un répit tout textuel, qui lui permet de vivre tant que la mort de Durdat n'a pas été racontée. Du vertige de la fiction, on passe au vertige du récit : celui-ci n'apparait plus comme un simple compte rendu des bouleversements du temps diégétique, ne fait pas que répercuter en son espace propre les conséquences d'une temporalité non linéaire, mais participe de plainpied à ce déphasage généralisé du temps et de la vitesse.

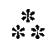

Dans "Man In His Time" de Brian Aldiss ${ }^{22}$, les lois de la conversation semblent temporairement suspendues: questions et réponses ne correspondent pas les unes aux autres; des questions surgissent que rien n'appelle, et auxquelles personne ne répond. Si le lecteur a tôt fait d'identifier le personnage responsable

22 Brian W. Aldiss, "Man In His Tïme", dans Man In His Time. Best SF Stories of Brian Alcliss, Londres, Victor Gollancz (coll. "VGSF"), 1989, p. 147-170. 


\section{2}

de ces perturbations, Jack Westermark, l'explication de son curieux comportement conversationnel, elle, se dérobe: désordre mental, effet d'une drogue quelconque, goût un peu puéril pour le nonsense? Rien de tout cela: Westermark, seul survivant d'une expédition désastreuse vers la planète Mars, vit maintenant dans une sorte de porte-à-faux temporel: il est "en avance" d'environ trois minutes trente sur la temporalité ambiante. S'il sursaute, c'est parce qu'un verre se brisera en éclats trois minutes trente plus tard; s'il dit "I'm sure we won't", commettant ainsi un quiproquo apparent, c'est qu'on demandera, toujours trois minutes trente plus tard, "Do you mind if I say goodbye to Nurse Simmons?"23. Le récit sollicitera les aptitudes cognitives du lecteur, par exemple lorsqu'un saignement de nez de Westermark résultera d'une situation qu'en même temps il déclenche (ou, plus exactement: résulte d'une situation qu'il déclenchera), provoquant ainsi un paradoxe temporel classique. Il le fait cependant moins sur le mode de l'exposition didactique que par la mise à plat de deux temporalités décalées - celle de Westermark et celle des autres personnages - en un seul récit, à la fois rigoureusement linéaire et cléchiré en chacune de ses articulations.

$$
\text { 隹济 }
$$

Vitesse de Westermark, lenteur de Westermark: sa perception précècle - comme on le dit d'une voiture filant devant nous sur l'autoroute - celle des autres personnages; mais son corps et ses réactions demeurent simultanés par rapport à ceux des autres personnages (du moins de leur point de vue). Le geste qu'il pose maintenant (dans ce qui constitue pour lui le "maintenant"), il l'a déjà posé (pour les autres personnages, dont les réactions sont,

23 Il faut réfléchir un peu pour saisir l'innovation que cela constitue. Alors que les récits classiques de voyage temporel propulsent leurs personnages respectifs clans un autre temps qui devient, pour la durée de leur séjour, leur temps, "Man in His 'lime "fait coexister les deux temporalités - la temporalité "normalen et celle de Westermark -, de sorte que ce dernier n'habitc, à proprement parler, aucun temps. Ou, plus exactement, son corps (et ses parcles, et ses mouvements) appartient à un "présent déjà révolu par rapport à celui cu'il est en train de percevoir. Ou, inversement: le "présent " qui soffie à sa perception relève, pour les autres personnages, d'un futur çui n'adviendra c fue trois minutes trente plus tard. 
pour lui, vieilles de trois minutes trente). On peut dès lors être tenté de voir dans "Man in His Time" une allégorie de la sciencefiction, ou plus précisément du récit d'anticipation, qui n'accomplit de projection imaginaire vers le futur qu'en injectant ce futur dans le présent de la lecture. À vouloir aller plus vite que les récits "réalistes", qui s'en tiennent au présent ou au passé, les écrivains de science-fiction finissent, comme le Seethwright de $L a$ dimension des miracles, par contribuer chacun à l'usure du continuum spatio-temporel, ou plutôt à son incessante perturbation. Que celle-ci ressortisse de l'imaginaire n'enlève rien au trouble et aux plaisirs - dans lesquels la science-fiction peut nous plonger. 